\title{
Running couplings for the simultaneous decoupling of heavy quarks
}

\author{
Steven D. Bass ${ }^{\mathrm{a}, *, 1}$, R.J. Crewther ${ }^{\mathrm{b}}$, F.M. Steffens ${ }^{\mathrm{c}, \mathrm{d}, 2}$, A.W. Thomas ${ }^{\mathrm{e}, 3}$ \\ ${ }^{a}$ Institute for Theoretical Physics, Universität Innsbruck, Technikerstrasse 25, A-6020 Innsbruck, Austria \\ ${ }^{\mathrm{b}}$ Department of Physics and Centre for the Subatomic Structure of Matter (CSSM), University of Adelaide, SA 5005, Australia \\ c Instituto de Fisica Teorica, UNESP, Rua Pamplona 145, 01405-900 São Paulo, SP, Brazil \\ d Mackenzie Presbiteriana University, FCBEE, Rua da Consolação 930, 01302-907 São Paulo, SP, Brazil \\ e Jefferson National Laboratory, 12000 Jefferson Avenue, Newport News, VA 23606, USA
}

Received 26 July 2005; received in revised form 12 January 2006; accepted 20 January 2006

Available online 3 February 2006

Editor: H. Georgi

\begin{abstract}
Scale-invariant running couplings are constructed for several quarks being decoupled together, without reference to intermediate thresholds. Large-momentum scales can also be included. The result is a multi-scale generalization of the renormalization group applicable to any order. Inconsistencies in the usual decoupling procedure with a single running coupling can then be avoided, e.g., when cancelling anomalous corrections from $t, b$ quarks to the axial charge of the proton.
\end{abstract}

(c) 2006 Elsevier B.V. All rights reserved.

PACS: $11.10 . \mathrm{Hi} ; 12.38 . \mathrm{Aw} ; 12.38 . \mathrm{Cy} ; 12.39 . \mathrm{Hg}$

Keywords: Renormalization group; Thresholds; Mass logarithms; Matching conditions; Axial anomaly

Normally, heavy quarks are decoupled one at a time, in order of decreasing mass $m_{q}, q=t, b, \ldots$. First the top quark is removed, with the $b$ - and $c$-quark thresholds left intact. Then $b$ is decoupled without destroying the $c$-quark threshold, and so on:

$m_{t} \rightarrow \infty$ first, then $m_{b} \rightarrow \infty, \quad$ then $m_{c} \rightarrow \infty$.

This sequential approach has two basic problems:

(1) On a logarithmic scale, $m_{t}$ may be sufficiently large compared with $m_{b}$, but not ${ }^{4} m_{b}$ compared with $m_{c}$.

\footnotetext{
* Corresponding author.

E-mail address: sbass@mail.cern.ch (S.D. Bass).

1 Supported by the Austrian Science Fund, contract P17778-N08.

2 Supported by FAPESP (03/10754-0) and CNPq (308932/2003-0).

3 Supported by DOE contract DE-AC05-84ER40150, under which SURA operates Jefferson National Laboratory.

4 The $t$ quark, the $W^{ \pm}$and $Z^{0}$ bosons, and (presumably) the Higgs boson, provide another example of this problem. When more than one of these particles sets the scale, or there are momenta of similar magnitude, it is not reasonable
}

(2) More seriously, it is inconsistent to retain both $O\left(\bar{\alpha}\left(m_{t}\right)\right)$ and $O\left(\bar{\alpha}\left(m_{b, c}\right)\right)$ corrections when the first step $m_{t} \rightarrow \infty$ assumes $\bar{\alpha}\left(m_{t}\right) \ll \bar{\alpha}\left(m_{b, c}\right)$; here $\bar{\alpha}(M)$ is the usual running coupling at scale $M$.

Our proposal is to replace the standard procedure by a new technique based on an extended renormalization group ( $R G)$ where heavy-quark decoupling is genuinely simultaneous and all mass logarithms are summed together. We show the need for this with an explicit example: anomaly cancellation for $t, b$ quarks decoupled from the axial charge of the proton.

We let two or more mass logarithms grow large together relative to a light-quark scale $\bar{\mu}$, e.g.,

$\ln \left(m_{c} / \bar{\mu}\right) \lesssim \ln \left(m_{b} / \bar{\mu}\right) \lesssim \ln \left(m_{t} / \bar{\mu}\right) \rightarrow$ large

for three heavy quarks. These heavy quarks are decoupled together, i.e., in a single step, without reference to intermediate thresholds. Instead of a single running coupling $\bar{\alpha}(M)$, there

to suppose that one of the corresponding logarithms dominates the others. See [1-5] for alternative methods. 
are separate running couplings $\alpha_{t}, \alpha_{b}, \ldots$, one for each heavy quark $h=t, b, \ldots$. The leading asymptotic power of an amplitude $\mathcal{A}$ is expanded as a multinomial in $\alpha_{t}, \alpha_{b}, \ldots$,

$\mathcal{A}=\sum_{k \ell \ldots} \alpha_{t}^{k} \alpha_{b}^{\ell} \cdots \mathcal{A}_{k \ell \ldots}$

with all $\left\{m_{h}\right\}$ dependence contained in $\alpha_{t}, \alpha_{b}, \ldots$. The coefficients $\mathcal{A}_{k \ell} \ldots$ are amplitudes produced by the residual lightquark theory. They can be identified by observing that the special case

$\ln \left(m_{t} / \bar{\mu}\right) \gg \ln \left(m_{b} / \bar{\mu}\right) \gg \cdots$

of the simultaneous limit (2) must give the same results as a direct application of the sequential limit (1). This technique depends on the fact that the residual theory is the same, irrespective of how the heavy quarks are decoupled.

A key step is the construction of simultaneous running couplings $\alpha_{t}, \alpha_{b}, \ldots$ for the general limit (2). Each $\alpha_{h}$ is RG invariant, depends on all of the mass logarithms in Eq. (2), and in the limit (4), can be expanded in running couplings $\tilde{\alpha}_{t}, \tilde{\alpha}_{b}$, and (if needed) $\tilde{\alpha}_{c}$ associated with separate steps in the sequential limit (1).

Let $\alpha_{f}=g_{f}^{2} / 4 \pi$ and $m_{q f}$ be the strong coupling and quark masses renormalized in the presence of $f$ flavours and three colours according to the $\overline{\mathrm{MS}}$ (modified minimal subtraction) scheme. We need the functions

$\beta_{f}(x)=-\frac{x^{2}}{6 \pi}(33-2 f)-\frac{x^{3}}{12 \pi^{2}}(153-19 f)+O\left(x^{4}\right)$,
$\delta_{f}(x)=-\frac{2 x}{\pi}+O\left(x^{2}\right)$

associated with the Callan-Symanzik operator

$\mathcal{D}_{f}=\bar{\mu} \frac{\partial}{\partial \bar{\mu}}+\beta_{f}\left(\alpha_{f}\right) \frac{\partial}{\partial \alpha_{f}}+\delta_{f}\left(\alpha_{f}\right) \sum_{q=1}^{f} m_{q f} \frac{\partial}{\partial m_{q f}}$,

where $\bar{\mu}=\sqrt{4 \pi} e^{-\gamma / 2} \mu_{\operatorname{dim}}$ is the $\overline{\mathrm{MS}}$ scale $\left(\mu_{\mathrm{dim}}\right.$ being the scale for dimensional renormalization). Note that $\bar{\mu}$ is chosen to be independent of $f$, that it sets the fixed low-energy scale needed to define simultaneous limits, as in Eq. (2), and that the heavy-quark masses for these limits are renormalized in the sixflavour theory:

$m_{h} \equiv m_{h 6}, \quad h=t, b, \ldots$

The running coupling $\alpha_{t}$ for the heaviest quark $t$ is defined by adapting Witten's prescription [6] for a single heavy quark. We set $f=6$,

$\ln \left(m_{t} / \bar{\mu}\right)=\int_{\alpha_{6}}^{\alpha_{t}} d x \frac{1-\delta_{6}(x)}{\beta_{6}(x)}$

and require that $\alpha_{6}$ be matched directly to the coupling $\alpha_{f}$ of the residual $f$-flavour theory in the limit (2). By construction, $\alpha_{t}$ is scale invariant with respect to the original $f=6$ theory:

$\mathcal{D}_{6} \alpha_{t}=0$.
Scale-invariant definitions of the other running couplings are then easily produced. The idea is to exploit a characteristic feature of simultaneous decoupling - the presence of large scale-invariant logarithms such as ${ }^{5}$

$\ln \left(m_{t} / m_{b}\right)=\ln \left(m_{t} / \bar{\mu}\right)-\ln \left(m_{b} / \bar{\mu}\right)$.

This logarithm can be used to set the relative scale between $\alpha_{t}$ and $\alpha_{b}$,

$\ln \left(m_{t} / m_{b}\right)_{6}=\int_{\alpha_{b}}^{\alpha_{t}} d x \frac{1-\delta_{5}(x)}{\beta_{5}(x)}$,

with $\alpha_{c}$ defined similarly if the $c$ quark is also being decoupled $^{6}$ :

$\ln \left(m_{b} / m_{c}\right)_{6}=\int_{\alpha_{c}}^{\alpha_{b}} d x \frac{1-\delta_{4}(x)}{\beta_{4}(x)}$.

Note that $\alpha_{b}$ and $\alpha_{c}$ exhibit the full $f=6$ scale invariance of the original theory

$\mathcal{D}_{6} \alpha_{b}=0=\mathcal{D}_{6} \alpha_{c}$

irrespective of the integrands chosen in Eqs. (12) and (13).

We will show that these definitions produce simultaneous running couplings $\alpha_{t}, \alpha_{b}, \ldots$ with the property

$\alpha_{h}=\underset{\text { seq }}{=} \tilde{\alpha}_{h}+O\left(\tilde{\alpha}_{t, b, \ldots}^{3}\right)$

in the sequential limit (4), where $\tilde{\alpha}_{t}, \tilde{\alpha}_{b}, \ldots$ are Witten's running couplings for successive steps in Eq. (1):

$\ln \left(m_{t 6} / \bar{\mu}\right)=\int_{\alpha_{6}}^{\tilde{\alpha}_{t}} d x \frac{1-\delta_{6}(x)}{\beta_{6}(x)}$,

$\ln \left(m_{b 5} / \bar{\mu}\right)=\int_{\alpha_{5}}^{\tilde{\alpha}_{b}} d x \frac{1-\delta_{5}(x)}{\beta_{5}(x)}$

$\ln \left(m_{c 4} / \bar{\mu}\right)=\int_{\alpha_{4}}^{\tilde{\alpha}_{b}} d x \frac{1-\delta_{4}(x)}{\beta_{4}(x)}$.

Eq. (15) says that $\alpha_{h}$ and $\tilde{\alpha}_{h}$ coincide asymptotically in leading order (LO) and next-to-leading order (NLO). ${ }^{7}$ LO agreement ensures that any amplitude $\mathcal{A}$ which can be expanded in the sequential limit is analytic in $\left\{\alpha_{h}\right\}$ when expanded in the simultaneous limit, as foreshadowed in Eq. (3), while agreement to NLO accuracy is convenient for practical purposes.

\footnotetext{
5 In the standard procedure (1), one chooses a scale for each step such that large logarithms never appear [7]. For simultaneous limits (2), that is no longer possible.

6 These definitions make sense for all mass values consistent with $m_{t} \geqslant m_{b} \geqslant$ $m_{c}$. This includes equal-mass cases, where running couplings coincide, e.g., $\alpha_{t}=\alpha_{b}$ for $m_{t}=m_{b}$.

7 For $\alpha_{t}$ and $\tilde{\alpha}_{t}$, the agreement is to all orders in the leading power: the defining equations (9) and (16) coincide.
} 
For example, let us decouple $t, b$ simultaneously from an amplitude $\mathcal{A}$. We match the simultaneous expansion (3) of $\mathcal{A}$ to terms produced by sequential decoupling, where first the top quark is decoupled with $m_{b}$ held fixed,

$\mathcal{A}=\mathcal{A}_{0}+\tilde{\alpha}_{t} \mathcal{A}_{1}+\tilde{\alpha}_{t}^{2} \mathcal{A}_{2}+O\left(\tilde{\alpha}_{t}^{3}\right)$

and then $b$ is decoupled from each of the residual five-flavour coefficient amplitudes $\mathcal{A}_{k}, k=0,1,2, \ldots$, to produce fourflavour amplitudes $\mathcal{A}_{k \ell}$ :

$\mathcal{A}_{k}=\mathcal{A}_{k 0}+\tilde{\alpha}_{b} \mathcal{A}_{k 1}+\tilde{\alpha}_{b}^{2} \mathcal{A}_{k 2}+O\left(\tilde{\alpha}_{b}^{3}\right)$.

Combine Eqs. (19) and (20), collecting terms of the same power in $\tilde{\alpha}_{t, b}$ :

$$
\begin{aligned}
\mathcal{A}= & \mathcal{A}_{00}+\left\{\tilde{\alpha}_{t} \mathcal{A}_{10}+\tilde{\alpha}_{b} \mathcal{A}_{01}\right\} \\
& +\left\{\tilde{\alpha}_{t}^{2} \mathcal{A}_{20}+\tilde{\alpha}_{t} \tilde{\alpha}_{b} \mathcal{A}_{11}+\tilde{\alpha}_{b}^{2} \mathcal{A}_{02}\right\}+O\left(\tilde{\alpha}_{t, b}^{3}\right) .
\end{aligned}
$$

Then, for any fixed value of $\ln \left(m_{t} / \bar{\mu}\right) / \ln \left(m_{b} / \bar{\mu}\right)$ from 1 to infinity, the linear (LO) and quadratic (NLO) corrections for $s i$ multaneous decoupling involve the same coefficient amplitudes as in Eq. (21), because of the property (15):

$$
\begin{aligned}
\mathcal{A} \underset{\operatorname{sim}}{=} & \mathcal{A}_{00}+\left\{\alpha_{t} \mathcal{A}_{10}+\alpha_{b} \mathcal{A}_{01}\right\} \\
& +\left\{\alpha_{t}^{2} \mathcal{A}_{20}+\alpha_{t} \alpha_{b} \mathcal{A}_{11}+\alpha_{b}^{2} \mathcal{A}_{02}\right\}+O\left(\alpha_{t, b}^{3}\right) .
\end{aligned}
$$

Coefficient amplitudes for higher orders can be calculated directly from cubic and higher-order corrections to Eqs. (15) and (21). Phenomenology based on single-limit expansions like (22) is perfectly consistent because all running couplings are of the same order, e.g., $\alpha_{t} \sim \alpha_{b}$.

The proof of Eq. (15) depends on RG equations which relate the initial $F$-flavour and residual $f$-flavour theories. These equations are equivalent to the rule [8] $\mathcal{D}_{F}=\mathcal{D}_{f}$ when these operators act on $f$-flavour quantities. Applied to $\alpha_{f}$ and $\ln m_{q f}$, this rule yields Witten's relations [6], generalized to include simultaneous cases (2):

$\mathcal{D}_{F} \alpha_{f}=\beta_{f}\left(\alpha_{f}\right)$,

$\mathcal{D}_{F} \ln m_{q f}=\delta_{f}\left(\alpha_{f}\right)$ for all $f<F$.

An immediate consequence of Eq. (23) is that the sequential running couplings defined by Eqs. (16)-(18) are all $\mathrm{RG}_{F=6}$ invariant [8]:

$\mathcal{D}_{6} \tilde{\alpha}_{t}=\mathcal{D}_{6} \tilde{\alpha}_{b}=\mathcal{D}_{6} \tilde{\alpha}_{c}=0$.

The proof also depends on matching conditions [8-12] for the $f=4,5,6$ couplings and masses in Eqs. (16)-(18). Let the decoupling of $h$ correspond to reducing the number of flavours from $f+1$ to $f$. Then a scale-invariant matching function $\mathcal{F}_{f+1 \rightarrow f}$ can be defined by the condition [8]

$$
\int_{\alpha_{f+1}}^{\tilde{\alpha}_{h}} \frac{d x}{\beta_{f+1}(x)}=\int_{\alpha_{f}}^{\tilde{\alpha}_{h}} \frac{d x}{\beta_{f}(x)}+\mathcal{F}_{f+1 \rightarrow f}\left(\tilde{\alpha}_{h}\right) .
$$

It contributes at NNLO (next-to-NLO):
$\mathcal{F}_{f+1 \rightarrow f}\left(\tilde{\alpha}_{h}\right)=-\frac{11}{12 \pi(33-2 f)} \tilde{\alpha}_{h}+O\left(\tilde{\alpha}_{h}^{2}\right)$.

There is also an invariant mass-matching function $\mathcal{G}_{f+1 \rightarrow f}$,

$$
\begin{aligned}
& \ln \frac{m_{q(f+1)}}{m_{q f}} \\
&=\int_{\alpha_{f}}^{\tilde{\alpha}_{h}} d x \frac{\delta_{f}(x)}{\beta_{f}(x)}-\int_{\alpha_{f+1}}^{\tilde{\alpha}_{h}} d x \frac{\delta_{f+1}(x)}{\beta_{f+1}(x)}+\mathcal{G}_{f+1 \rightarrow f}\left(\tilde{\alpha}_{h}\right)
\end{aligned}
$$

which contributes at NNNLO (next-to-NNLO) [8]:

$\mathcal{G}_{f+1 \rightarrow f}\left(\tilde{\alpha}_{h}\right)=-\frac{89}{432 \pi^{2}} \tilde{\alpha}_{h}^{2}+O\left(\tilde{\alpha}_{h}^{3}\right)$.

Now let Eqs. (12), (16), (17) and the identity

$\ln \left(m_{t} / m_{b}\right)_{6}=\ln \left(m_{t 6} / \bar{\mu}\right)-\ln \left(m_{b 5} / \bar{\mu}\right)-\ln \left(m_{b 6} / m_{b 5}\right)$

be combined with Eqs. (25) and (27) for the case $f=5, h=t$. The result is a relation between the various running couplings for $t$ and $b$ :

$$
\begin{aligned}
& \int_{\tilde{\alpha}_{t}}^{\alpha_{t}} d x \frac{1-\delta_{5}(x)}{\beta_{5}(x)} \\
& \underset{\text { seq }}{=} \int_{\tilde{\alpha}_{b}}^{\alpha_{b}} d x \frac{1-\delta_{5}(x)}{\beta_{5}(x)}+\mathcal{F}_{6 \rightarrow 5}\left(\tilde{\alpha}_{t}\right)-\mathcal{G}_{6 \rightarrow 5}\left(\tilde{\alpha}_{t}\right) .
\end{aligned}
$$

The $b, c$ running couplings are similarly related:

$$
\begin{array}{rl}
\int_{\tilde{\alpha}_{b}}^{\alpha_{b}} & d x \\
& =\frac{1-\delta_{4}(x)}{\beta_{4}(x)} \\
& =\int_{\text {seq }}^{\alpha_{c}} d x \frac{1-\delta_{4}(x)}{\beta_{4}(x)}+\mathcal{F}_{5 \rightarrow 4}\left(\tilde{\alpha}_{b}\right)-\mathcal{G}_{5 \rightarrow 4}\left(\tilde{\alpha}_{b}\right) .
\end{array}
$$

Eq. (15) is an immediate consequence of Eqs. (30) and (31): the matching functions do not contribute at NLO, and we have ${ }^{7}$ $\alpha_{t}=\tilde{\alpha}_{t}$ for the top couplings. Indeed, the NNLO corrections are easily found:

$\alpha_{b}=\tilde{\alpha}_{\text {seq }}-\frac{11}{72 \pi^{2}} \tilde{\alpha}_{b}^{2} \tilde{\alpha}_{t}+O\left(\tilde{\alpha}_{t, b}^{4}\right)$,

$\alpha_{c}=\tilde{\alpha}_{c}-\frac{11}{72 \pi^{2}} \tilde{\alpha}_{c}^{2}\left(\tilde{\alpha}_{b}+\tilde{\alpha}_{t}\right)+O\left(\tilde{\alpha}_{t, b, c}^{4}\right)$.

As noted below Eq. (9), $\alpha_{6}$ and $m_{q 6}$ have to be matched directly to their counterparts in the residual $f$-flavour theory. This is done in two steps: we replace Witten's invariant mass [6] $\tilde{m}_{h}$ for the heavy quark $h$ in Eq. (25)

$$
\ln \left(\tilde{m}_{h} / \bar{\mu}\right)=\int_{\alpha_{f+1}}^{\tilde{\alpha}_{h}} \frac{d x}{\beta_{f+1}(x)}
$$

with a set of simultaneous $\mathrm{RG}_{F=6}$ invariant masses $\bar{m}_{t}, \bar{m}_{b}, \ldots$, and then define simultaneous matching functions $\mathcal{F}_{6 \rightarrow f}$ and $\mathcal{G}_{6 \rightarrow \mathrm{f}}$. 
In the case of simultaneous $t, b$ decoupling, where the residual theory has four flavours, $\bar{m}_{t}$ and $\bar{m}_{b}$ are defined by the equations

$\ln \left(\bar{m}_{t} / \bar{\mu}\right)=\int_{\alpha_{6}}^{\alpha_{t}} \frac{d x}{\beta_{6}(x)}$,

$\ln \left(\bar{m}_{t} / \bar{m}_{b}\right)=\int_{\alpha_{b}}^{\alpha_{t}} \frac{d x}{\beta_{5}(x)}$.

Then $\mathcal{F}_{6 \rightarrow 4}$ is defined by the condition

$\ln \left(\bar{m}_{b} / \bar{\mu}\right)=\int_{\alpha_{4}}^{\alpha_{b}} \frac{d x}{\beta_{4}(x)}+\mathcal{F}_{6 \rightarrow 4}\left(\alpha_{t}, \alpha_{b}\right)$.

Being $\mathrm{RG}_{F=6}$ invariant, $\mathcal{F}_{6 \rightarrow 4}$ can depend only on $\alpha_{t}$ and $\alpha_{b}$. The matching condition between $\alpha_{6}$ and $\alpha_{4}$ for simultaneous $t, b$ decoupling is the result of eliminating $\alpha_{t}$ and $\alpha_{b}$ from Eqs. (35) and (36).

An alternative definition of $\mathcal{F}_{6 \rightarrow 4}$ is obtained by eliminating $\bar{m}_{t}$ and $\bar{m}_{b}$ from Eqs. (35) and (36):

$$
\begin{aligned}
\mathcal{F}_{6 \rightarrow 4}\left(\alpha_{t}, \alpha_{b}\right) & \\
= & -\int_{\alpha_{4}}^{\alpha_{b}} d x \frac{1}{\beta_{4}(x)}-\int_{\alpha_{b}}^{\alpha_{t}} d x \frac{1}{\beta_{5}(x)}-\int_{\alpha_{t}}^{\alpha_{6}} d x \frac{1}{\beta_{6}(x)} .
\end{aligned}
$$

Comparison with Eq. (27) then suggests the following $\mathrm{RG}_{F=6}$ invariant definition of a simultaneous mass-matching function $\mathcal{G}_{6 \rightarrow 4}:$

$$
\begin{aligned}
\ln \frac{m_{\ell 6}}{m_{\ell 4}}= & \int_{\alpha_{4}}^{\alpha_{b}} d x \frac{\delta_{4}(x)}{\beta_{4}(x)}+\int_{\alpha_{b}}^{\alpha_{t}} d x \frac{\delta_{5}(x)}{\beta_{5}(x)} \\
& +\int_{\alpha_{t}}^{\alpha_{6}} d x \frac{\delta_{6}(x)}{\beta_{6}(x)}+\mathcal{G}_{6 \rightarrow 4}\left(\alpha_{t}, \alpha_{b}\right) .
\end{aligned}
$$

Eqs. (37) and (38) can be matched to Eqs. (25)-(28) and (32) in the sequential limit (4) to determine the leading terms in $\mathcal{F}_{6 \rightarrow 4}$ (NNLO) and $\mathcal{G}_{6 \rightarrow 4}$ (NNNLO):

$$
\begin{aligned}
& \mathcal{F}_{6 \rightarrow 4}=-\frac{11}{300 \pi}\left(\alpha_{b}+\alpha_{t}\right)+O\left(\alpha_{b, t}^{2}\right), \\
& \mathcal{G}_{6 \rightarrow 4}=-\frac{89}{432 \pi^{2}}\left(\alpha_{b}^{2}+\alpha_{t}^{2}\right)-\frac{11}{1725 \pi^{2}} \alpha_{b} \alpha_{t}+O\left(\alpha_{b, t}^{3}\right) .
\end{aligned}
$$

For simultaneous $t, b, c$ decoupling, we add

$\ln \left(\bar{m}_{b} / \bar{m}_{c}\right)=\int_{\alpha_{c}}^{\alpha_{b}} \frac{d x}{\beta_{4}(x)}$

to the definitions (35) and specify matching functions $\mathcal{F}_{6 \rightarrow 3}$ (NNLO) and $\mathcal{G}_{6 \rightarrow 3}$ (NNNLO) as follows:

$\ln \frac{\bar{m}_{c}}{\bar{\mu}}=\int_{\alpha_{3}}^{\alpha_{c}} d x \frac{1}{\beta_{3}(x)}+\mathcal{F}_{6 \rightarrow 3}\left(\alpha_{t}, \alpha_{b}, \alpha_{c}\right)$,

$$
\begin{aligned}
\ln \frac{m_{\ell 6}}{m_{\ell 3}}= & \int_{\alpha_{3}}^{\alpha_{c}} d x \frac{\delta_{3}(x)}{\beta_{3}(x)}+\int_{\alpha_{c}}^{\alpha_{b}} d x \frac{\delta_{4}(x)}{\beta_{4}(x)}+\int_{\alpha_{b}}^{\alpha_{t}} d x \frac{\delta_{5}(x)}{\beta_{5}(x)} \\
& +\int_{\alpha_{t}}^{\alpha_{6}} d x \frac{\delta_{6}(x)}{\beta_{6}(x)}+\mathcal{G}_{6 \rightarrow 3}\left(\alpha_{t}, \alpha_{b}, \alpha_{c}\right) .
\end{aligned}
$$

The dependence of our running couplings on mass logarithms can be determined order by order from perturbative results for the Callan-Symanzik and matching functions. An example is the $\mathrm{LO}$ asymptotic behaviour of $\alpha_{t}$ for simultaneous $t, b, c$ decoupling:

$\alpha_{t} \sim 6 \pi /\left(23 \ln \left(m_{t} / \bar{\mu}\right)+2 \ln \left(m_{b} / \bar{\mu}\right)+2 \ln \left(m_{c} / \bar{\mu}\right)\right)$.

Generally, this coupling runs with a mixture of $f=3,4$, and 5 active flavours. The sequential limit (4) is a special case, where the dominant term in the denominator exhibits the five-flavour coefficient 23 ( $33-2 f$ for $f=5$ ). Another extreme case is $m_{t}=m_{b}$ with $\ln \left(m_{c} / \bar{\mu}\right)$ neglected, where the coefficient becomes $25(f=4)$. Similarly, we get the $f=3$ coefficient 27 for $m_{t}=m_{b}=m_{c}$.

The multi-scale RG can be readily generalized to include logarithmic momentum scales between the mass logarithms in Eq. (2). The main new feature is that the invariant masses given by Eqs. (35) and (40) are used to construct scale-invariant logarithms. For example, let $Q$ be a large momentum scale contained in the simultaneous limit

$\ln \left(m_{b} / \bar{\mu}\right) \lesssim \ln (Q / \bar{\mu}) \lesssim \ln \left(m_{t} / \bar{\mu}\right) \rightarrow$ large

Then amplitudes are expanded as multinomials in $\alpha_{t}, \alpha_{b}$ and $\alpha_{Q}$, where $\alpha_{Q}$ is defined by

$\ln \left(Q / \bar{m}_{b}\right)=\int_{\alpha_{b}}^{\alpha_{Q}} \frac{d x}{\beta_{5}(x)}$

or equivalently by

$\ln \left(\bar{m}_{t} / Q\right)=\int_{\alpha_{Q}}^{\alpha_{t}} \frac{d x}{\beta_{5}(x)}$.

This completes the formulation of the multi-scale RG. Its advantage over the standard method (1) is that to a given order, no logarithms are discarded, so uncontrolled approximations or outright contradictions (such as the example of anomaly cancellation below) are avoided. Each application of the standard method should be checked to see how well it approximates the multi-scale result.

The need for a multi-scale approach is best illustrated by the classic example [7,13-17] of heavy-quark decoupling from the weak neutral axial-vector current

$J_{\mu 5}^{Z}=\frac{1}{2}\left\{\sum_{q=u, c, t}-\sum_{q=d, s, b}\right\}\left(\bar{q} \gamma_{\mu} \gamma_{5} q\right)_{6}$,

where the subscript 6 indicates an operator or amplitude in QCD with $f=6$ flavours. Diagrams for LO contributions in ghost-free gauges are shown in Fig. 1. 


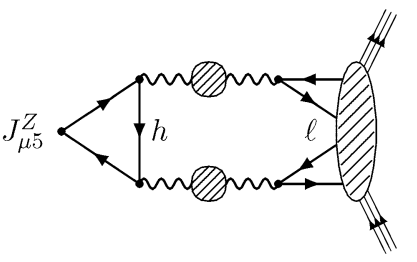

Fig. 1. Leading-order diagrams for heavy quarks $h$ to decouple from light quarks $\ell$ when the weak neutral current $J_{\mu 5}^{Z}$ is coupled to a nucleon at low momentum transfer. The gluon propagators (indicated by wavy lines) are dressed with multiple one-loop self-energy insertions.

Let us apply the standard procedure (1) to the six-flavour amplitude

$\Gamma_{\mu 5}^{(6)}=\left\langle\bar{t} \gamma_{\mu} \gamma_{5} t-\bar{b} \gamma_{\mu} \gamma_{5} b\right\rangle_{6}$

The first step $m_{t} \rightarrow \infty$ produces a current operator with scaleinvariant renormalization $[14,15]$ :

$\lim _{m_{t} \rightarrow \infty} \Gamma_{\mu 5}^{(6)}=\Gamma_{\mu 5}^{(5)}=-\left\langle\left(\bar{b} \gamma_{\mu} \gamma_{5} b\right)_{\text {inv }}\right\rangle_{5}$.

In the $\mathrm{LO}$ correction to this result,

$\Gamma_{\mu 5}^{(6)}-\Gamma_{\mu 5}^{(5)}=-\frac{6 \tilde{\alpha}_{t}}{23 \pi}\left\langle\sum_{q=u}^{b}\left(\bar{q} \gamma_{\mu} \gamma_{5} q\right)_{\mathrm{inv}}\right\rangle_{5}$

the Witten running coupling $\tilde{\alpha}_{t}$ displays a five-flavour dependence $[7,14]$ :

$\tilde{\alpha}_{t} \sim \frac{6 \pi}{23 \ln \left(m_{t} / \bar{\mu}\right)}$.

This agrees with the rule of thumb that the number of active flavours is determined by the residual theory.

The problem becomes more subtle when both $t$ and $b$ are to be decoupled such that anomaly cancellation occurs for $m_{t}=m_{b}$. The sequential method (1) requires us to decouple the $b$ quark from Eq. (49),

$\Gamma_{\mu 5}^{(6)} \underset{\text { LO seq }}{=} \frac{6}{23 \pi}\left(\tilde{\alpha}_{b}-\tilde{\alpha}_{t}\right)\left\langle\sum_{q=u}^{c}\left(\bar{q} \gamma_{\mu} \gamma_{5} q\right)_{\text {inv }}\right\rangle_{4}$

but it is immediately evident that the method fails with respect to cancellation at $m_{b}=m_{t}$ because $\tilde{\alpha}_{b}$ runs with four flavours:

$\tilde{\alpha}_{b} \sim \frac{6 \pi}{25 \ln \left(m_{t} / \bar{\mu}\right)}$.

Presumably this is why the sequential method was not pursued for this example in [7,14]; instead, a five-flavour dependence was proposed for both the $t$ and $b$ contributions:

$$
\begin{aligned}
\Gamma_{\mu 5}^{(6)} \stackrel{?}{\sim} & \left(\frac{6}{23}\right)^{2}\left\{\frac{1}{\ln \left(m_{b} / \bar{\mu}\right)}-\frac{1}{\ln \left(m_{t} / \bar{\mu}\right)}\right\} \\
& \times\left\langle\sum_{q=u}^{c}\left(\bar{q} \gamma_{\mu} \gamma_{5} q\right)_{\mathrm{inv}}\right\rangle_{4} .
\end{aligned}
$$

Certainly this formula vanishes for $m_{t}=m_{b}$, but the fiveflavour dependence assumed for both scales is not possible: the residual theory has only four flavours.
The multi-scale RG resolves these problems very simply. Eq. (51) is a double asymptotic series of the form (21) derived for the strictly sequential limit (1). One cannot apply it directly to the case $m_{t}=m_{b}$ because important LO logarithms have been discarded. To restore all logarithms, replace Witten's running couplings $\tilde{\alpha}_{t}, \tilde{\alpha}_{b}$ by our simultaneous couplings $\alpha_{t}, \alpha_{b}$ and so obtain a simultaneous expansion of the form (22):

$\Gamma_{\mu 5}^{(6)} \underset{\mathrm{LO} \operatorname{sim}}{=} \frac{6}{23 \pi}\left(\alpha_{b}-\alpha_{t}\right)\left\langle\sum_{q=u}^{c}\left(\bar{q} \gamma_{\mu} \gamma_{5} q\right)_{\mathrm{inv}}\right\rangle_{4}$.

Since ${ }^{6} \alpha_{t}=\alpha_{b}$ for $m_{t}=m_{b}$, anomaly cancellation is automatic; at the same time, this result includes the sequential formula (51) as a special case. These properties are especially evident in the asymptotic result replacing (53):

$$
\begin{aligned}
& \Gamma_{\mu 5}^{(6)} \underset{\operatorname{sim}}{\sim} \frac{36}{23}\left[\left\{25 \ln \frac{m_{b}}{\bar{\mu}}\right\}^{-1}-\left\{23 \ln \frac{m_{t}}{\bar{\mu}}+2 \ln \frac{m_{b}}{\bar{\mu}}\right\}^{-1}\right] \\
& \times\left\langle\sum_{q=u}^{c}\left(\bar{q} \gamma_{\mu} \gamma_{5} q\right)_{\text {inv }}\right\rangle_{4} .
\end{aligned}
$$

A direct analysis of the diagrams in Fig. 1 gives the same answer [16].

The next order in the simultaneous expansion can be deduced from the NLO correction to the sequential result (51) found in [15]. Making the replacement $\tilde{\alpha}_{t, b} \rightarrow \alpha_{t, b}$, we find:

$$
\begin{aligned}
& \Gamma_{\mu 5}^{(6)}= \frac{6}{\mathrm{NLO}}\left(\alpha_{b}-\alpha_{t}\right)\left\{1+\frac{125663}{82800 \pi} \alpha_{b}+\frac{6167}{3312 \pi} \alpha_{t}\right\} \\
& \times\left\langle\sum_{q=u}^{c}\left(\bar{q} \gamma_{\mu} \gamma_{5} q\right)_{\text {inv }}\right\rangle_{4} .
\end{aligned}
$$

The factor $\alpha_{b}-\alpha_{t}$ ensures that anomaly cancellation occurs automatically at $m_{t}=m_{b}$.

In practice, the multi-scale RG will be important in determining momentum dependence between heavy-quark thresholds, e.g., in deep inelastic scattering. It will allow the whole range of momenta, from one threshold to the next, to be described by a single expansion calculable (in principle) to any order.

When a threshold is crossed, the multi-scale asymptotic expansion changes because the relevant operator-product and heavy-quark expansions differ. Consequently there are slope discontinuities at thresholds, as for other mass-independent methods [18]. The standard prescription for the removal of discontinuities is the method of effective charges [5,19-21], but this has been set up only for the single-scale RG. Formulating a natural multi-scale extension of this method presents an interesting challenge for the future.

The result of this Letter is a substantial generalization of the RG which solves problems involving logarithms for more than one large scale. Amplitudes are expanded as multinomials of running couplings, one for each large scale but depending (in general) on all of these scales; this replaces the familiar perturbation series in one running coupling for a single-scale RG. All multi-scale results to a given order (degree in the multinomial 
expansion) can be readily deduced from single-scale calculations to the same order. Our method avoids inconsistencies by retaining all important logarithms to a given order.

\section{References}

[1] F.V. Tkachov, Phys. Lett. B 412 (1997) 350;

M. Beneke, V.A. Smirnov, Nucl. Phys. B 522 (1998) 321;

V.A. Smirnov, Applied Asymptotic Expansions in Momenta and Masses, Springer, Berlin, 2002.

[2] J. Blumlein, W.L. van Neerven, Phys. Lett. B 450 (1999) 417.

[3] W. Beenakker, S. Dittmaier, M. Krämer, B. Plümper, M. Spira, P.M. Zerwas, Phys. Rev. Lett. 87 (2001) 201805; L. Reina, S. Dawson, D. Wackeroth, Phys. Rev. D 65 (2002) 053017.

[4] A. Denner, in: Int. Europhysics Conference on High Energy Physics, JHEP Proc. PrHEP hep2001/129, Budapest, 2001, hep-ph/0110155; S. Pozzorini, Nucl. Phys. B 692 (2004) 135.

[5] S.J. Brodsky, M.S. Gill, M. Melles, J. Rathsman, Phys. Rev. D 58 (1998) 116006;

M. Binger, S.J. Brodsky, Phys. Rev. D 69 (2004) 095007.

[6] E. Witten, Nucl. Phys. B 104 (1976) 445.

[7] J. Collins, F. Wilczek, A. Zee, Phys. Rev. D 18 (1978) 242.

[8] S.D. Bass, R.J. Crewther, F.M. Steffens, A.W. Thomas, Phys. Rev. D 68 (2003) 096005.
[9] W. Bernreuther, W. Wetzel, Nucl. Phys. B 197 (1982) 228;

W. Bernreuther, W. Wetzel, Nucl. Phys. B 513 (1998) 758, Erratum; W. Bernreuther, Ann. Phys. (N.Y.) 151 (1983) 127.

[10] S.A. Larin, T. van Ritbergen, J.A.M. Vermaseren, Nucl. Phys. B 438 (1995) 278

[11] K.G. Chetyrkin, B.A. Kniehl, M. Steinhauser, Phys. Rev. Lett. 79 (1997) 2184;

K.G. Chetyrkin, B.A. Kniehl, M. Steinhauser, Nucl. Phys. B 510 (1998) 61.

[12] G. Rodrigo, A. Pich, A. Santamaria, Phys. Lett. B 424 (1998) 367.

[13] D.B. Kaplan, A.V. Manohar, Nucl. Phys. B 310 (1988) 527.

[14] K.G. Chetyrkin, J.H. Kühn, Z. Phys. C 60 (1993) 497.

[15] S.D. Bass, R.J. Crewther, F.M. Steffens, A.W. Thomas, Phys. Rev. D 66 (2002) 031901(R).

[16] R.J. Crewther, S.D. Bass, F.M. Steffens, A.W. Thomas, Nucl. Phys. B (Proc. Suppl.) 141 (2005) 159.

[17] S.D. Bass, Rev. Mod. Phys. 77 (2005) 1257.

[18] W.J. Marciano, Phys. Rev. D 29 (1984) 580.

[19] G. Grunberg, Phys. Rev. D 29 (1984) 2315.

[20] D.C. Kennedy, B.W. Lynn, Nucl. Phys. B 322 (1989) 1.

[21] D.V. Shirkov, I.L. Solovtsov, Phys. Rev. Lett. 79 (1997) 1209; D.V. Shirkov, I.L. Solovtsov, Phys. Lett. B 442 (1998) 344. 